\title{
Adverse events following vaccination in the French armed forces: An overview of surveillance conducted from 2002 to 2010
}

\author{
A Mayet (aurelie_marie@hotmail.fr) ${ }^{1}$, R Haus-Cheymol ${ }^{1}$, E A Bouaiti ${ }^{1}$, C Decam $^{2}$, F Simon ${ }^{3}$, A Mérens $^{4}$, A Spiegel ${ }^{1,5}$, J B Meynard M $^{1,5}$, \\ X Deparis ${ }^{2,5}$, R Migliani ${ }^{1,5}$ \\ 1. Centre d'épidémiologie et de santé publique des armées, Saint Mandé, France \\ 2. Centre d'épidémiologie et de santé publique des armées, Institut de médecine tropicale du Service de santé des armées, \\ Marseille, France \\ 3. Service de pathologie infectieuse et tropicale, Hôpital d'instruction des armées Laveran, Marseille, France \\ 4. Fédération de biologie clinique, Hôpital d’instruction des armées Bégin, Saint Mandé, France \\ 5. École du Val-de-Grâce, Paris, France
}

Citation style for this article:

Mayet A, Haus-Cheymol R, Bouaiti EA, Decam C, Simon F, Mérens A, Spiegel A, Meynard JB, Deparis X, Migliani R. Adverse events following vaccination in the French armed forces: An overview of surveillance conducted from 2002 to 2010. Euro Surveill. 2012;17(24):pii=20193. Available online: http://www.eurosurveillance.org/

ViewArticle.aspx?Articleld $=20193$

Article submitted on 13 December 2011 / published on 14 June 2012

French military personnel are subject to a compulsory vaccination schedule. The aim of this study was to describe vaccine adverse events (VAE) reported from 2002 to 2010 in armed forces. VAE are routinely surveyed by the military Centre for epidemiology and public health. For each case, military practitioners fill a notification form, providing patient characteristics, clinical information and vaccines administered. For this study, VAE following influenza $A\left(\mathrm{H}_{1} \mathrm{~N}_{1}\right)$ pdmo9 vaccination were excluded. Among the 473 cases retained, 442 (93\%) corresponded to non-severe VAE, including local, regional and systemic events, while 31 corresponded to severe VAE, with two leading to significant disability. The global VAE reporting rate (RR) was 14.0 per 100,000 injections. While stationary from 2002 to 2008, the RR increased from 2009. The most important observations were a marked increase of VAE attributed to Bacillus Calmette-Guérin (BCG) vaccine from 2005 to 2008 , a high RR observed with the inactivated diphtheria-tetanus (toxoids)-poliovirus vaccine combined with acellular pertussis vaccine (dTap-IPV) from 2008 and an increase in RR for seasonal influenza vaccine VAE in 2009. Our RR for severe VAE (1.1 VAE per 100,000) appears comparable with rates observed among United States civilians and military personnel. The increase observed from 2009 could be partly explained by the influenza $A\left(\mathrm{H}_{1} \mathrm{~N}_{1}\right)$ pdmog pandemic which increased practitioner awareness towards VAE. In conclusion, the tolerance of the vaccines used in French armed forces appears acceptable.

\section{Introduction}

Due to their collective lifestyle and their operational imperatives, military personnel are exposed to infections that they can contract during training or overseas missions and that can be prevented by vaccination [1-4]. Thus, according to a vaccination strategy that targets individual and collective protection, French military personnel are subject to a compulsory vaccination schedule at enlistment and during their whole service period (Figure 1). This schedule implies the administration of vaccines that are often injected simultaneously: Bacillus Calmette-Guérin vaccine (BCG), inactivated diphtheria-tetanus (toxoids)-poliovirus vaccine (dT-IPV), which can be also combined with acellular pertussis vaccine (dTap-IPV), inactivated influenza vaccine, $A_{C Y W_{135}}$ polysaccharide meningococcal vaccine, subunit hepatitis $B$ vaccine, whole virus inactivated hepatitis $A$ vaccine, typhoid vaccine, live yellow fever vaccine and measles-mumps-rubella vaccine (MMR). Most of these vaccines are administered during the two-month period following enrollment, taking into account previously administered vaccines. Certain specific vaccinations are individualised according to occupational imperatives (e.g. rabies, leptospirosis, tick-borne encephalitis). The vaccination schedule is reconsidered each year and updated according to main epidemiological events.

The French military health department that implements this vaccination schedule is also responsible for the vaccine adverse event (VAE) surveillance network in the French armed forces. The objectives of this network are to detect previously unrecognised reactions to current vaccines as well as unusual increases in reported VAE [5]. This article presents the results of VAE surveillance in the French armed forces from 2002 to 2010.

\section{Methods}

VAE in the armed forces have been under surveillance since 2002 by the Centre for epidemiology and public health (Centre d'épidémiologie et de santé publique des armées or (ESPA) of the French military health department. The military epidemiological surveillance is mandatory and concerns all active military personnel (average of 342,337 personnel-years between 
Compulsory vaccination schedule at enlistment for French military personnel likely to be deployed after six month of service, France, 2012

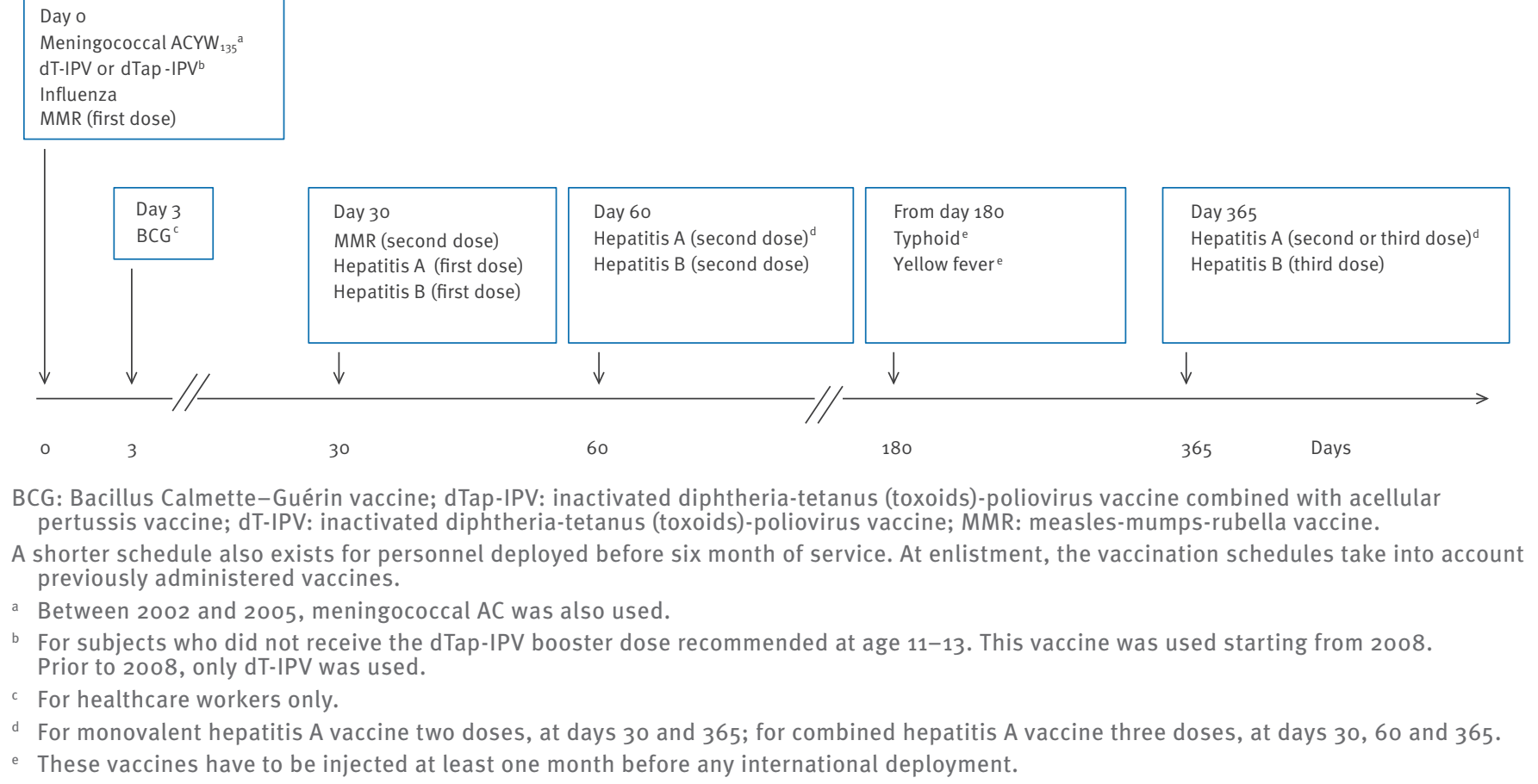

03

30

60

From day 180

Typhoide

Yellow fever ${ }^{\mathrm{e}}$

Day 365

Hepatitis A (second or third dose)

Hepatitis B (third dose)

BCG: Bacillus Calmette-Guérin vaccine; dTap-IPV: inactivated diphtheria-tetanus (toxoids)-poliovirus vaccine combined with acellular pertussis vaccine; dT-IPV: inactivated diphtheria-tetanus (toxoids)-poliovirus vaccine; MMR: measles-mumps-rubella vaccine.

A shorter schedule also exists for personnel deployed before six month of service. At enlistment, the vaccination schedules take into account previously administered vaccines.

a Between 2002 and 2005, meningococcal AC was also used.

b For subjects who did not receive the dTap-IPV booster dose recommended at age 11-13. This vaccine was used starting from 2008. Prior to 2008 , only dT-IPV was used.

For healthcare workers only.

d For monovalent hepatitis A vaccine two doses, at days 30 and 365; for combined hepatitis A vaccine three doses, at days 30,60 and 365 .

e These vaccines have to be injected at least one month before any international deployment.

2002 and 2010, with 35,000 personnel enrolled each year). Every week, military physicians are required to complete a form which mentions the number of cases observed for some monitored health events, including VAE. For each suspected VAE case, the practitioner who sees the patient completes another form providing information concerning the patient, the clinical symptoms and the vaccines administered. The forms are transmitted weekly to the CESPA which analyses the data after checking. Practitioners who sent an incomplete form are re-contacted by the CESPA for data completion. All VAE report forms are then routinely sent to the French Health Products Safety Agency. Cases are classified on the basis of clinical description derived from French drug vigilance guidelines [6]. Two types of VAE are considered:

- Non-severe adverse events: VAE following vaccination, which can be: (i) local (e.g. pain, lump at the injection point, redness $>5 \mathrm{~cm}$ or other local events like pruritus or haematoma) that persist at least 48 hours; (ii) regional (e.g. ulcer, lymph node tenderness and/or enlargement, adenitis, abscess at the injection site); (iii) systemic (fever $\geq 38^{\circ} \mathrm{C}$ or any event thought to be related to vaccination, with sick leave for more than two days);

- Severe adverse events: VAE with hospitalisation, persistent or significant disability, life-threatening illness or death. This category has to be reported immediately to the CESPA and requires a review of medical charts.
Whether severe or non-severe, VAE that are not listed in the French Summary of Product Characteristics (SPC) are also considered as unexpected VAE.

For this study, systemic events that did not lead to a sick leave of more than two days and local events that persisted less than 48 hours were excluded from analyses. VAE following vaccinations with the monovalent $\mathrm{A}\left(\mathrm{H}_{1} \mathrm{~N}_{1}\right)$ pdmo9 vaccine, reported between 2009 and 2010, were also excluded. This is because the influenza $A\left(\mathrm{H}_{1} \mathrm{~N}_{1}\right)$ pdmog vaccination campaign constituted an exceptional phenomenon, which was difficult to compare with what is usually observed in terms of VAE. This data has been previously published [5].

In case of simultaneous administration of several vaccines, the following vaccine suspicion algorithm was used: (i) for local or regional VAE, the vaccine suspected was the vaccine administered at the site of VAE occurrence; (ii) for systemic or severe VAE, all vaccines administered were suspected.

The use of this algorithm explains that the number of suspected vaccines exceeded the number of VAE. Moreover, a vaccination could be responsible of several simultaneous VAE in a same subject (for example, fever associated with a lump at the injection point). For this reason, the number of VAE presented exceeds the number of cases initially reported. 
The analysis concerned the 2002-2010 period. The reporting rates for VAE cases were calculated by dividing the number of VAE by the number of military personnel monitored (rates per 100,000 person-years). The vaccine-specific VAE reporting rates (rates per 100,000 vaccinations) were calculated by dividing the number of VAE following a specific vaccine (according to the vaccine suspicion algorithm), by the number of doses distributed according to the French military drug supply department for the same specific vaccine. Reporting rates were compared using negative binomial regressions (model controlled on year for vaccinespecific rates and multivariate model controlled on year, sex and age for VAE cases). Data analysis was performed using Stata version 9.

\section{Results}

\section{Vaccine-specific reporting rate}

for vaccine adverse events

From 2002 to 2010, 798 cases of VAE were reported, of which $170(21 \%)$ were excluded because they did not meet case report criteria. After also excluding the 155 VAE following $A\left(\mathrm{H}_{1} \mathrm{~N}_{1}\right)$ pdmog vaccination, the analyses were performed on 473 VAE cases who presented 634 VAE. After applying the vaccine suspicion algorithm, 681 vaccine injections could be suspected in the occurrence of these VAE and were used for the vaccine-specific reporting rate calculations.
The global VAE reporting rate for the 2002-2010 period was 13.6 VAE per 100,000 injections $(681 / 4,991,270)$. While the VAE reporting rates did not vary from 2002 to 2008 (reporting rates ranging from 7.9 to 13.7 per 100,000 injections), a significant increase was observed in 2009 and 2010 (respectively 20.7 and 24.9 per 100,000, p 0.001 ). As shown in Table 1, the dTap-IPV vaccine, used in the French armed forces only since 2008 , had the highest global VAE reporting rate of all vaccines considered for the 2002-2010 period (107.2 per 100,000). Among VAE following BCG vaccination (second highest global rate for the 2002-2010 period: 62.8 per 100,000 ), the multipuncture vaccine (Monovax), used from 2002 to 2005 in armed forces, accounted for a 46.0 per 100,000 reporting rate while the intradermal vaccine (BCG SSI), used from 2006 to 2010 , accounted for a 564.0 per 100,000 reporting rate. The dTap-IPV vaccine had the highest reporting rates for local reactions ( 45.6 per 100,000), systemic reactions ( 49.6 per 100,000) and severe VAE (2.7 per $100,000)$. The BCG vaccine had the highest reporting rate for regional reactions (34.8 per 100,000).

No VAE following MMR vaccination, which was used from 2008 in the French armed forces, was reported despite increasing use on account of the recent measles epidemic $(9,471$ doses injected in 2010) [2].

In terms of evolution of VAE across the period, the most important observations (Figure 2) were: (i) the very high rates in VAE following BCG vaccination from

TABLE 1

Vaccine-specific reporting rates for vaccine adverse events per 100,000 vaccinations reported in French armed forces according to vaccine suspicion algorithm, France, 2002-2010

\begin{tabular}{|c|c|c|c|c|c|c|c|c|c|c|c|c|}
\hline \multirow{2}{*}{ Vaccines } & \multicolumn{2}{|c|}{ Total VAE } & \multicolumn{2}{|c|}{ Local VAE } & \multicolumn{2}{|c|}{ Regional VAE } & \multicolumn{2}{|c|}{ Systemic VAE } & \multicolumn{2}{|c|}{ Severe VAE } & \multicolumn{2}{|c|}{ Unexpected VAE } \\
\hline & $\mathrm{N}$ & Rate ${ }^{a}$ & $\mathrm{~N}$ & Rate $^{\mathrm{a}}$ & $\mathrm{N}$ & Rate $^{\mathrm{a}}$ & $\mathrm{N}$ & Rate $^{\mathrm{a}}$ & $\mathrm{N}$ & Rate $^{a}$ & $\mathrm{~N}$ & Rate $^{\mathrm{a}}$ \\
\hline BCG (Monovax, SSI) ${ }^{b}$ & 65 & 62.8 & 26 & 25.1 & 36 & 34.8 & 1 & 1.0 & 2 & 1.9 & 0 & 0.0 \\
\hline dTap-IPV (Repevax ) & 80 & 107.2 & 34 & 45.6 & 7 & 9.4 & 37 & 49.6 & 2 & 2.7 & 2 & 2.7 \\
\hline dT-IPV (Revaxis) & 75 & 16.7 & 36 & 8.0 & 6 & 1.3 & 27 & 6.0 & 6 & 1.3 & 0 & 0.0 \\
\hline Hepatitis A and B (Twinrix) & 26 & 11.2 & 9 & 3.9 & 2 & 0.9 & 14 & 6.1 & 1 & 0.4 & 1 & 0.4 \\
\hline Hepatitis A (Havrix 1440) & 31 & 6.0 & 3 & 0.6 & 2 & 0.4 & 20 & 3.9 & 6 & 1.2 & 0 & 0.0 \\
\hline Hepatitis B (Engerix B) & 15 & 9.0 & 4 & 2.4 & 2 & 1.2 & 8 & 4.8 & 1 & 0.6 & 1 & 0.6 \\
\hline $\begin{array}{l}\text { Influenza (Influvac, Mutagrip, } \\
\text { Vaxigrip) }\end{array}$ & 159 & 13.2 & 50 & 4.1 & 19 & 1.6 & 79 & 6.6 & 11 & 0.9 & 2 & 0.2 \\
\hline Meningococcal AC (Pasteur) ${ }^{c}$ & 39 & 12.0 & 15 & 4.6 & 10 & 3.1 & 10 & 3.1 & 4 & 1.2 & 1 & 0.3 \\
\hline $\begin{array}{l}\text { Meningococcal } \mathrm{ACYW}_{135} \\
\text { (Menomune, Mencevax) }\end{array}$ & 77 & 11.0 & 28 & 4.0 & 8 & 1.1 & 31 & 4.4 & 10 & 1.4 & 3 & 0.4 \\
\hline Typhoid (Typhim Vi, Typhérix) & 43 & 5.6 & 4 & 0.5 & o & 0.0 & 34 & 4.4 & 5 & 0.6 & 2 & 0.3 \\
\hline Yellow fever (Stamaril) & 71 & 15.8 & 6 & 1.3 & 16 & 3.6 & 43 & 9.6 & 6 & 1.3 & 0 & 0.0 \\
\hline Total & 681 & 13.6 & 215 & 4.3 & 108 & 2.2 & 304 & 6.1 & 54 & 1.1 & 12 & 0.2 \\
\hline
\end{tabular}

BCG: Bacillus Calmette-Guérin vaccine; dTap-IPV: inactivated diphtheria-tetanus (toxoids)-poliovirus vaccine combined with acellular pertussis vaccine; dT-IPV: inactivated diphtheria-tetanus (toxoids)-poliovirus vaccine; VAE: vaccine adverse event.

a The rate is given per 100,000 vaccinations.

b Monovax was replaced by SSI from 2006.

Meningococceal AC vaccine was only used from 2002 to 2004 . 
Evolution of respective vaccine adverse events rates reported in French armed forces for seasonal influenza, Bacillus Calmette-Guérin and dTap-IPV vaccines, France, 2002-2010

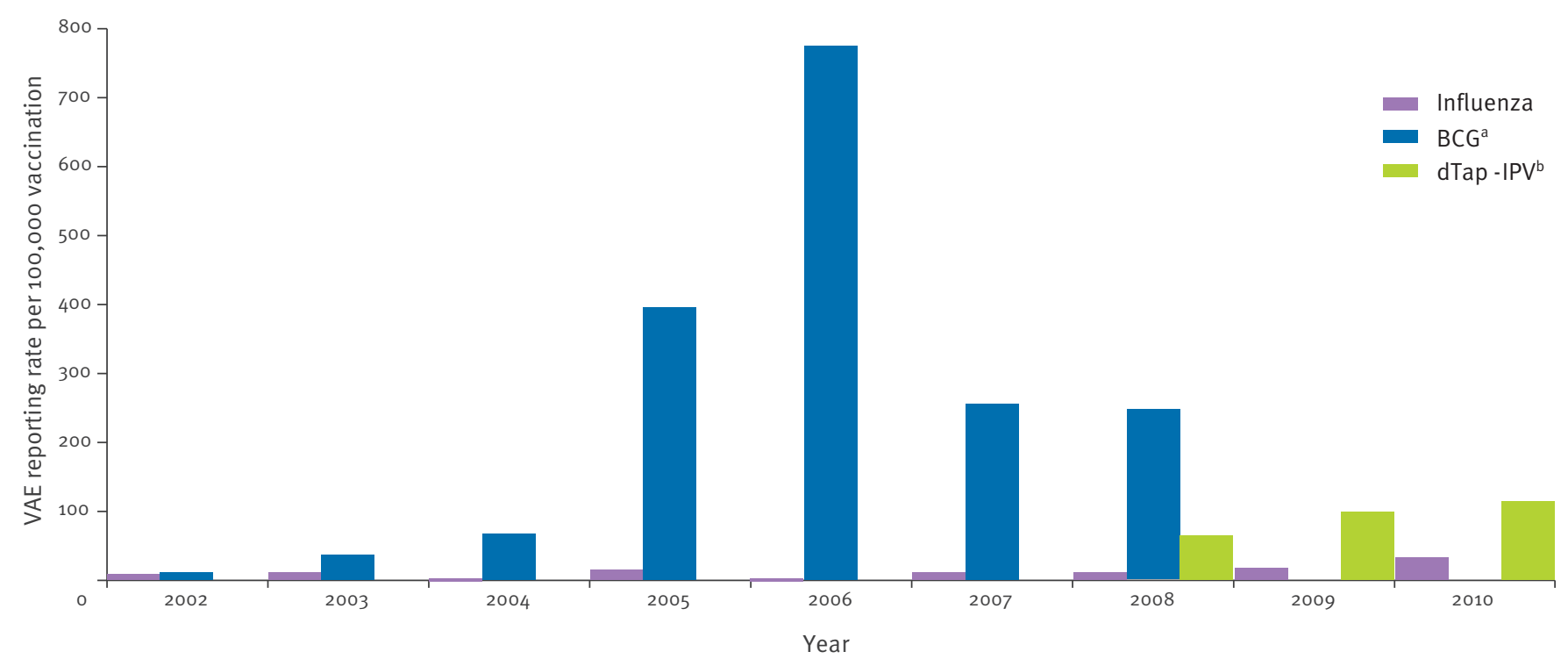

BCG: Bacillus Calmette-Guérin; dTap-IPV: inactivated diphtheria-tetanus (toxoids)-poliovirus vaccine combined with acellular pertussis vaccine; VAE: vaccine adverse events.

a BCG multipuncture vaccine (Monovax) was replaced by BCG intradermal vaccine (SSI) in 2006.

b dTap-IPV was used starting 2008.

2005 to 2008 ( $p<0.001$ ), the reporting rate reaching 785.2 VAE per 100,000 vaccinations in 2006; (ii) the high reporting rates observed with the dTap-IPV vaccine from the beginning of its use, with a tendency to increase between 2008, 2009 and 2010 (respectively $65.5,100.3$ and 115.7 VAE per 100,000 vaccinations; $\mathrm{p}=0.2$ ); (iii) an increase in seasonal influenza vaccine VAE reporting rates from 2009 (17.9 VAE per 100,000 in 2009 and 32.8 VAE per 100,000 vaccinations in 2010; $\mathrm{p}=0.05)$.

Characteristics of vaccine adverse events cases Among the 473 military personnel who presented a VAE, 142 (30\%) were women and 213 (45\%) belonged to the Army. The median age of cases was 26 years (interquartile range (IQR) [21-33]). Multivariate analysis showed a 1.9 times greater risk of VAE among women (33.5 versus 12.5 cases per 100,000 persons-years among men; p<0.001). In addition, being under 20 yielded a 15.7 times greater risk of VAE than being 50 and older ( 94.6 versus 5.5 cases per 100,000 personsyears; $p<0.001)$.

\section{Severity of the vaccine adverse events}

Among the 473 cases reported, 93\% were non-severe. These 442 cases corresponded to $603 \mathrm{VAE}$, given the associations observed in 150 cases (34\%) of several types of VAE. Two hundred and forty-nine cases presented a local VAE (56\%), 123 (28\%) presented a regional VAE and 231 (52\%) presented a systemic VAE (Figure 3). Local VAE were mainly characterised by pain
(38\%), redness (35\%) and lump at the injection point (25\%). Clinical features of systemic VAE frequently included an influenza-like syndrome most often with fever, arthralgia and headaches. The mean time lapse from vaccination to the occurrence of the VAE was 2.6 days for local VAE (median: 0.7, IQR: 0.2-1.2 days), 8.4 days for regional VAE (median: 1.1, IQR: 0.4-6.0 days) and 1.6 days for systemic VAE (median: 0.8, IQR: 0.31.9 days).

Only $31 \operatorname{VAE}(7 \%)$, corresponding to the same number of cases, were considered as severe because they led to hospitalisation (Table 2). The mean time lapse from vaccination to the occurrence of the severe VAE was 26.2 days (median: 2.0, IQR: 1.0-6.0 days). The maximal time-lapse (563.0 days) was observed for a macrophagic myofasciitis following hepatitis $A$ vaccination. Neurological syndromes were predominantly severe headaches (7 cases) which sometimes occurred in a context of meningeal-like syndrome (3 cases). One subject developed acute leucoencephalomyelitis three weeks after seasonal influenza vaccination, leading to cognitive and sensory sequelae. Still's disease, a rheumatic disorder, occurred in a case aged 20 who had received yellow fever and hepatitis $A$ vaccines the same day. A few hours after vaccination, the patient presented transitory dysesthesia in the median nerve area of the limb where the yellow fever vaccine had been injected. Two months later, this case presented a polyarthritis which led to significant lack of mobility. The BCG vaccine was responsible for two severe 
Frequency of vaccine adverse events $(n=603)$ reported by French military personnel (n=442), France, 2002-2010

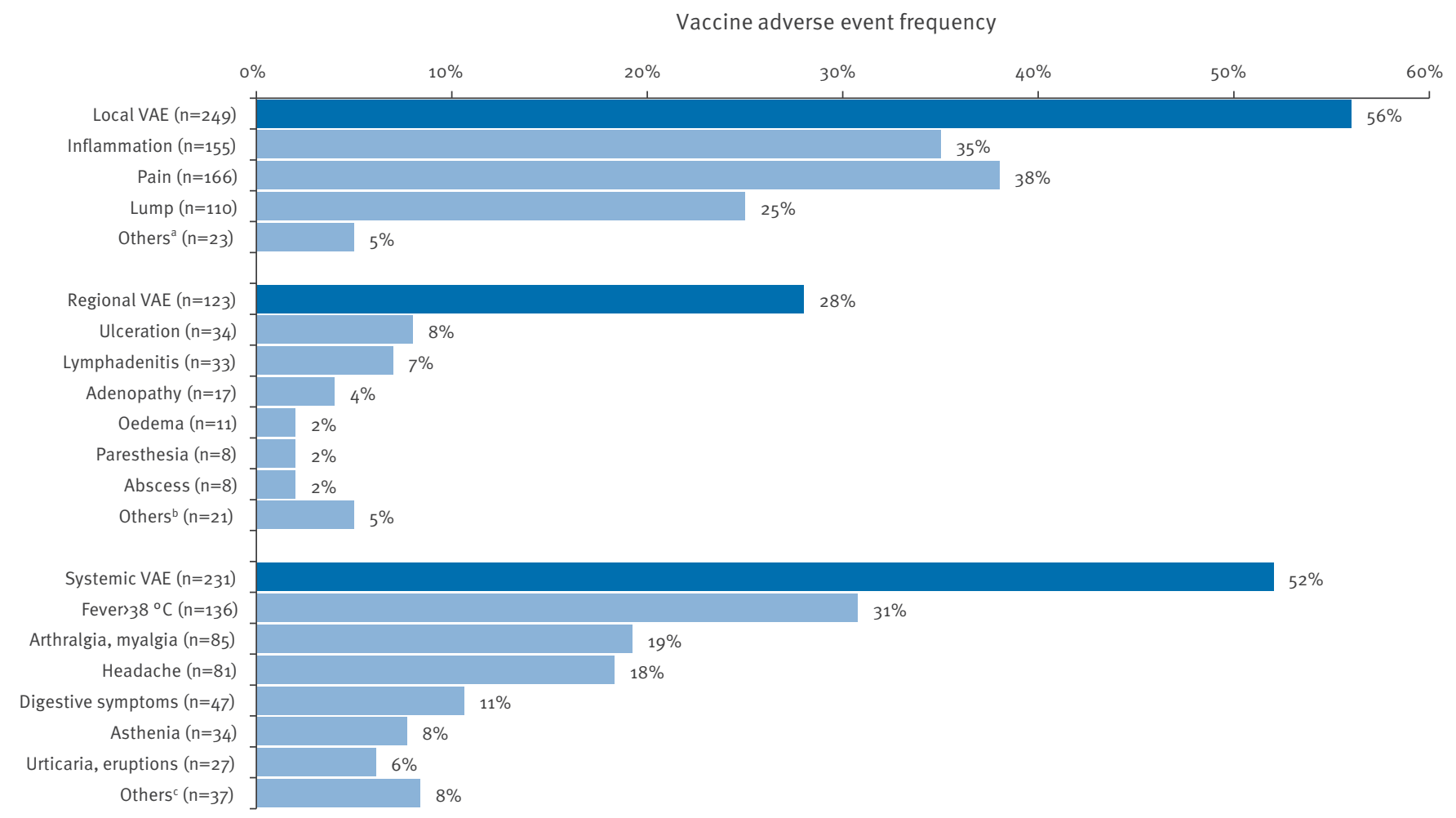

VAE: vaccine adverse event.

a Refers to other types of local vaccine adverse events.

b Refers to other types of regional vaccine adverse events.

c Refers to other types of systemic vaccine adverse events.

local ulcerations, probably aggravated by poor hygiene during a training period occurring after vaccination. The three cases of urticaria reported as severe VAE were not life-threatening but were treated and monitored in hospital, unlike the urticaria cases reported as non-severe VAE which were treated in the medical department of the military unit. This situation was also observed for headaches and influenza-like syndromes that were reported as severe VAE. Finally, although the outcome of the macrophagic myofasciitis is unknown, all other reported severe VAE, with the exception of the cases of leucoencephalomyelitis and Still's disease, regressed without sequelae.

Only nine unexpected VAE were reported ( $2 \%$ ), including ear, nose and throat disorders (ENT) symptoms (epistaxis, hypoacusis tinnitus), ophthalmological symptoms (temporary loss of visual acuteness, central chorioretinitis), one case of myopericarditis (which was also considered as a severe VAE), one of psoriasis, and one of monoplegia of the vaccinated limb.

\section{Discussion}

Reporting the vaccine adverse events The strength of this study is that it provides recent data concerning VAE for a nine-year period in a healthy, high-vaccinated and adult (18-65 age stratum) population. As most of the vaccinations administered in armed forces are compulsory and military personnel can consult a physician in the medical department that performed the vaccine injection, it is likely that our data could be representative of the majority of VAE that occurred in this population. The high exclusion rate observed over the study period $(21 \%)$ accounts to the reliability of our data: if so many patients consulted for minor symptoms, there is likely that the surveillance system would not miss real VAE cases. However, the use of a passive reporting system may expose to under-reporting from some practitioners.

Given the absence of reliable data concerning doses injected, the number of doses distributed was chosen as denominator for vaccine-specific rate calculations, 
Clinical features and administered vaccines for cases of severe vaccine adverse events $(\mathrm{n}=31)$ reported in French armed forces, France, 2002-2010

\begin{tabular}{|c|c|c|c|c|}
\hline Type of VAE & $\begin{array}{c}\text { Symptoms } \\
\text { (number of cases) }\end{array}$ & $\begin{array}{c}\text { Sick leave } \\
\text { length in } \\
\text { days }\end{array}$ & $\begin{array}{l}\text { Time- } \\
\text { lapse from } \\
\text { injection to } \\
\text { VAE in days }\end{array}$ & Vaccines administered ${ }^{a}$ \\
\hline \multirow[t]{2}{*}{ Local VAE } & Ulceration $(n=1)$ & 16 & 73 & BCG \\
\hline & Abscess $(n=1)$ & 1 & 2 & BCG \\
\hline \multirow[t]{11}{*}{ Neurological syndromes } & \multirow[t]{2}{*}{ Headache $(n=2)$} & 1 & 2 & Meningococcal AC, dT-IPV, typhoid \\
\hline & & 2 & NN & $\begin{array}{l}\text { Meningococcal } \mathrm{ACYW}_{135} \text {, yellow fever, typhoid, } \\
\text { influenza }\end{array}$ \\
\hline & \multirow[t]{3}{*}{ Meningeal-like syndrome $(n=3)$} & 3 & 3 & Meningococcal AC, dT-IPV \\
\hline & & 2 & 2 & Yellow fever \\
\hline & & NN & 1 & Meningococcal $\mathrm{ACYW}_{135}$, hepatitis $\mathrm{A}$ \\
\hline & \multirow[t]{2}{*}{ Headache and vertigo $(n=2)$} & 2 & 1 & Yellow fever, hepatitis A, typhoid \\
\hline & & 1 & 5 & Yellow fever, dT-IPV \\
\hline & Cerebellar syndrome $(\mathrm{n}=1)$ & 18 & 1 & Hepatitis A, meningococcal AC \\
\hline & Obnubilation $(n=1)$ & 1 & 1 & dT-IPV \\
\hline & Leucoencephalomyelitis $(\mathrm{n}=1)$ & 180 & 22 & Influenza \\
\hline & Monoplegia of vaccinated limb $(n=1)$ & NN & 5 & Hepatitis A and B, typhoid \\
\hline \multirow[t]{5}{*}{ Metabolic syndromes } & \multirow[t]{3}{*}{ Thrombopenia/bleeding $(n=3)$} & NN & 10 & Influenza, hepatitis B \\
\hline & & 20 & 6 & Meningococcal ACYW ${ }_{135}$, influenza \\
\hline & & 20 & 6 & Meningococcal $\mathrm{ACYW}_{135}$, influenza \\
\hline & Renal insufficiency $(n=1)$ & NN & 3 & Influenza, yellow fever \\
\hline & Hypoglycemia $(n=1)$ & NN & 2 & Influenza \\
\hline \multirow[t]{13}{*}{ Miscellaneous syndromes } & \multirow[t]{3}{*}{ Urticaria $(n=3)$} & 1 & 1 & Influenza \\
\hline & & 3 & 0 & Hepatitis A \\
\hline & & 1 & 0 & Meningococcal ACYW ${ }_{135}$, typhoid \\
\hline & Macrophagic myofasciitis $(n=1)$ & NN & 563 & Hepatitis A \\
\hline & \multirow[t]{4}{*}{ Influenza-like syndrome $(n=4)$} & 3 & 0 & $\mathrm{dT}$-IPV, meningococcal AC \\
\hline & & NN & 0 & Typhoid \\
\hline & & 13 & 6 & Meningococcal ACYW ${ }_{135}$, dT-IPV \\
\hline & & 4 & 1 & Meningococcal ACYW ${ }_{135}$, dTap-IPV \\
\hline & Myopericarditis $(n=1)$ & 7 & 2 & Influenza \\
\hline & \multirow[t]{2}{*}{ Vagal malaise $(\mathrm{n}=2)$} & 1 & 0 & Influenza, meningococcal ACYW ${ }_{135}$, dTap-IPV \\
\hline & & 2 & 1 & Influenza \\
\hline & Still's disease $(n=1)$ & 116 & 60 & Yellow fever, hepatitis A \\
\hline & Spreading myalgia $(n=1)$ & 45 & 6 & Hepatitis A, meningococcal ACYW $_{135}$ \\
\hline
\end{tabular}

BCG: Bacillus Calmette-Guérin; dT-IPV: inactivated diphtheria-tetanus (toxoids)-poliovirus vaccine; dTap-IPV: inactivated diphtheria-tetanus (toxoids)-poliovirus vaccine combined with acellular pertussis vaccine; NN: not known; VAE: vaccine adverse event.

a In cases of vaccine association, the first vaccine of the list is the one that was initially suspected by the practitioner who performed the vaccination.

which could lead to an underestimation of VAE. However, this number may not be too different from the number of doses really injected if considering that military units, constrained by economic imperatives, order vaccines as and when required, keeping little stock which may expire.

VAE constitute a relatively rare phenomenon in the French armed forces if we consider the large number of vaccinations performed (around 500,000 each year). The reporting rate of 14 VAE per 100,000 injections observed in French armed forces appears higher than the rate (4 VAE per 100,000) observed in United
States (US) general population aged $18-65$ years for the 1991-2001 period [8]. Although non-severe VAE are not routinely monitored in the French civilian population, a study using the drug vigilance database of a pharmaceutical laboratory showed a reporting rate of 20 VAE per 100,000 for the 2000-2010 period, which could correspond to a 7 per 100,000 reporting rate among 18-65 year-olds if it is assumed that the distribution of VAE according to age is the same in France as in US [9]. The higher reporting rate observed in our study could reflect better reporting of non-severe VAE by military physicians, who could be more inclined to follow instructions to report VAE compared to civilian 
physicians, the French military surveillance system being compulsory and the subject of a training course. This hypothesis is also supported by the higher proportion of reported severe VAE in the US civilian data (14\% versus $7 \%$ among French military forces). Moreover, the severe VAE reporting rate among French armed forces (1.1 per 100,000) was comparable with rates observed among US civilians (0.6 per 100,000) and US military personnel for the 1998-2002 period (0.8 per 100,000 for yellow fever vaccine and 1.3 per 100,000 for typhoid vaccine) [10]. However, these cross-national comparisons in reporting rates are problematic due to differing populations, reporting procedures and exclusion criteria.

The highest reporting rate, when considering the whole period studied, was observed with the dTap-IPV vaccine. As this vaccine has been used only since 2008 in the French armed forces, this high reporting rate could reflect the fact that military physicians perceived dTapIPV as a "new" vaccine, leading to a tendency to more complete reporting [11]. This more complete reporting, which may not be the consequence of diagnosis biases, could lead to more representative rates even if they are unusually high. The dTap-IPV vaccine is also known to be implicated in certain non-severe VAE. A study conducted among US healthcare personnel in 2006 showed that $68 \%$ of vaccinated subjects reported an injection site reaction and $10 \%$ reported subjective fever [12]. However, although the higher severe VAE reporting rate also concerned the dTap-IPV vaccine in our data, the VAE reported were relatively benign. A study conducted from 2004 to 2008 concluded that the dTap-IPV vaccine has a similar safety profile to that of dT-IPV vaccine in terms of severe VAE [13].

High reporting rates were observed with the BCG vaccine for the study period, particularly with the intradermal vaccine. In the French general population, ulcerations have been reported to follow 1 to $2 \%$ of BCG vaccinations [14]. The peak in reported VAE observed in 2006 coincided with the withdrawal of multipuncture $B C G$ vaccine (Monovax) in France, replaced by the intradermal BCG vaccine (BCG SSI) [15]. Practitioners were not accustomed to the administration of this new vaccine, which could have involved some administration errors and led to the observed increase in VAE this year [16]. This led French health authorities to publish recommendations of good practice [17]. From 2007, BCG vaccination was limited to certain populations at risk and a decrease in number of injected doses occurred from this year in the French armed forces (387 in 2007 versus 1,165 in 2006).

Finally, an increase in reported seasonal influenza VAE occurred at the end of 2009. It mainly involved nonsevere events and probably reflects stimulated reporting in the context of the upcoming influenza $A\left(\mathrm{H}_{1} \mathrm{~N}_{1}\right)$ pdmog pandemic [18]. Subsequently, 155 VAE following pandemic influenza vaccination were reported in the French armed forces within a six-month period while VAE incidence for all other vaccines was only 45 cases for the same period, which is mainly explained by an over-reporting effect [5]. It is possible that this episode increased practitioner awareness towards VAE reporting, which could explain the increase in reporting observed in 2009 and 2010 while the VAE reporting rate was stationary between 2002 and 2008. The numerous VAE following dTap-IPV vaccination reported from 2008 may have also contributed to this observed increase.

Taking into account the number of military personnel monitored, the VAE reporting rate was the highest among subjects under 20 years of age ( 94.6 cases per 100,000 person-years). This age stratum corresponds to the personnel recently enlisted and consequently more likely to receive a number of vaccines due to the military vaccination schedule.

\section{Severity of vaccine adverse events}

Most of the VAE reported (93\%) were non-severe effects, which, in addition to the relatively low reporting rates observed, amounts to an acceptable tolerance of vaccines among French military personnel. Most of the 31 severe VAE reported between 2002 and 2010 were considered as severe only when hospitalisation of concerned cases occurred. For example, the three urticaria cases were only characterised by a diffuse superficial eruption, without impact on respiratory function or circulation, and regressed rapidly after anti-histaminic treatment. In addition, 27 other subjects presented a cutaneous eruption which fitted the characteristics of diffuse superficial urticaria. These cases were considered as non-severe VAE because they were treated in the medical department of the military unit and monitored for a few hours, without hospitalisation. It is true that the reporting rate for life-threatening anaphylactic reactions following vaccination is very low, ranging from one to three cases per million vaccinations [19]. Thus, the hospitalisation criterion in the definition of severe VAE does not always reflect the clinical severity of the case's status because the decision to hospitalise also depends on other factors: presence of a nearby medical facility, experience of the general practitioner who initially treats the case, or operational imperatives.

On a case by case basis, causal attribution of VAE to vaccines is scientifically difficult [20], particularly for rare or unexpected events, and this difficulty increases in case of simultaneous injection of several vaccines, which concerned $58 \%$ of severe VAE observed in our study (18/31). Finally, VAE cases constitute a minor phenomenon if compared to the many cases avoided by vaccination concerning some diseases. Significant decreases in meningococcal meningitis and hepatitis A incidences were thus observed following the implementation of systematic vaccination at enlistment in armed forces [21-23]. 


\section{Conclusion}

VAE appears to be relatively rare in French armed forces, particularly severe VAE. Our results are concordant with previous research and account for an acceptable tolerance of vaccines. The French armed forces, which enrol around 35,000 personnel and perform more than 500,000 vaccinations each year, are an important vaccine vigilance observatory in France while the surveillance of non-severe VAE is not compulsory in civilian population [24]. The monitoring of VAE remains topical in armed forces because it assures military personnel that safety of required vaccines is taken seriously and constitutes an indirect indicator of the acceptability of vaccination campaigns from patient and practitioner viewpoints.

\section{Acknowledgments}

Authors would like to thank all the military physicians and the staff of the Epidemiological and public health centre who participated actively in the military epidemiological surveillance network by way of the quality of their notifications and reports.

\section{References}

1. Debord T, Eono P, Rey JL, Roue R. [Infectious risks among military personnel deployed in operation]. Med Mal Infect. 1996;26 Suppl 3:402-7. French.

2. Mayet A, Verret C, Haus-Cheymol R, Duron S, De Laval F, Imbert $P$, et al. Resurgence of measles among French military forces in 2010. Eur J Microbiol Infect Dis. 2011;30(8):1023-6.

3. Mayet A, Brossier C, Haus-Cheymol R, Verret C, Meynard JB, Migliani R et al.. Pertussis surveillance within the French armed forces: a new system showing increased incidence among young adults (2007-2009). J Infect. 2011;62(4):322-4

4. Mayet A, Duron S, Nivoix P, Haus-Cheymol R, Ligier C, Gache $\mathrm{K}$, et al. Novel influenza $A\left(\mathrm{H}_{1} \mathrm{~N}_{1}\right)$ outbreak among French armed forces in 2009: Results of Military Influenza Surveillance System. Public Health. 2011;125(8):494-500.

5. Mayet A, Ligier C, Gache K, Manet G, Nivoix P, Dia A, et al. Adverse events following pandemic influenza vaccine Pandemrix $\square$ reported in the French military forces - 2009-2010 Vaccine. 2011;29(14):2576-81.

6. Begaud B, Chaslerie A, Haramburu F. [Organization and results of drug vigilance in France]. Rev Epidemiol Sante Publique. 1994;42(5):416-23. French.

7. Thiessard F, Roux E, Miremont-Salame G, Fourrier-Reglat A, Haramburu F, Tubert-Bitter $P$, et al. Trends in spontaneous adverse drug reaction reports to the French pharmacovigilance system (1986-2001). Drug Saf. 2005;28(8):731-40.

8. Zhou W, Pool V, Iskander JK English-Bullard R, Ball R, Wise RP, et al. Surveillance for safety after immunization: VAE Reporting System (VAERS)-United States, 1991-2001. MMWR Surveill Summ. 2003;52(1):1-24.

9. Okaïs C, Gay C, Seon F, Buchaille L, Chary E, Soubeyrand B. Disease-specific adverse events following nonlive vaccines: a paradoxical placebo effect or a nocebo phenomenon? Vaccine. 2011;29(37):6321-6.

10. Khromava AY, Eidex RB, Weld LH, Kohl KS, Bradshaw RD, Chen RT, et al. Yellow fever vaccine: an updated assessment of advanced age as a risk factor for serious adverse events. Vaccine. 2005;23(25):3256-63.

11. Weber JCP. Epidemiology of adverse reactions to nonsteroidal anti-inflammatory drugs. In: Rainsford KD, Velo GP, editors. Advances in Inflammation Research. Vol 6. New York: Raven Press; 1984:1-7.

12. Talbot EA, Brown KH, Kirkland KB, Baughman AL, Halperin SA, Broder KR. The safety of immunizing with tetanusdiphtheria-acellular pertussis vaccine (Tdap) less than 2 years following previous tetanus vaccination: Experience during a mass vaccination campaign of healthcare personnel during a respiratory illness outbreak. Vaccine. 2010;28(50):8001-7.
13. Yih WK, Nordin JD, Kulldorff M, Lewis E, Lieu TA, Shi P, et al. An assessment of the safety of adolescent and adult tetanusdiphtheria-acellular pertussis (Tdap) vaccine, using active surveillance for adverse events in the Vaccine Safety Datalink. Vaccine. 2009;27(32):4257-62

14. French health general authority. Guide des vaccinations [Guide of vaccinations]. Saint-Denis: Institut national de prevention et d'éducation pour la santé (INPES); 2008. French. [Accessed 11 Jun 2012]. Available from: http://www.inpes.sante.fr/10000/ themes/vaccination/guide_2008/pdf/GV2008.pdf

15. de la Rocque F, Cohen R, Vie le Sage F, Bocquet A, Boucherat $M$, Levy Bruhl D. [French paediatrician and general practitionner's survey about actual and future BCG use]. Arch Pediatr. 2005;12(11):1665-9. French.

16. Wattrelot $P$, Brion JP, Labarère J, Billette de Villemeur A, GirardBlanc MF, Stahl JP, et al. [Vaccination practices following the end of BCG compulsory vaccination. A cross sectional survey of general practitioners and pediatricians]. Arch Pediatr. 2010;17(2):118-24. French.

17. Effets indésirables au cours d'une vaccination par le vaccin BCG SSI@. 20/07/2006 [Adverse events following vaccination with $B C G$ SSI ${ }^{\circledR}$ vaccine, 20/07/2006]. Agence française pour la sécurité sanitaire des produits de santé (Afssaps). French. [Accessed 11 Jun 2012]. Available from: http://www.ansm.sante.fr/S-informer/PresseCommuniques-Points-presse/Effets-indesirables-au-coursd-une-vaccination-par-le-vaccin-BCG-SSI-R/(language)/ fre-FR

18. Mayet A, Nivoix $P$, Haus-Cheymol $R$, De Laval F, Verret $C$, Duron S, et al. Increase in reported adverse events following seasonal influenza vaccination among the French armed forces, 2008-2009: possible role of stimulated reporting and background cases of influenza-like infection. Public Health. 2012;126(1):70-6.

19. Zent O, Arras-Reiter C, Broeker M, Hennig R. Immediate allergic reactions after vaccinations - a post marketing surveillance review. Eur J Pediatr. 2002;161(1):21-5.

20. Stratton K, Ford A, Rusch E, Wright Clayton E, Editors; Committee to Review Adverse Effects of Vaccines et al. Adverse events of vaccines: evidence and causality. Washington, DC: The National Academies press; 2011. [Accessed 6 Sep 2011]. Available from: http://www.nap.edu/catalog. php?record id=13164\#toc

21. Buisson Y, Rey JL, Nicolas P, Soares JL, Meyran M. Vaccination systématique des militaires français contre les méningococcies $A$ et $C$. Bilan après 5 ans [Systematic vaccination of French military against $A$ and $C$ meningococcal meningitides: report after 5 years]. Bull Epidemiol Hebd. 1999;11:42-43. French.

22. Bradshaw RD. Meningococcal disease in the United States active duty military: historical experience, immunization, and current epidemiology 1999-2005. European congress of vaccinology in Armed forces; 2006 October 16-17; Paris, France. [Accessed 26 Sep 2011]. Available from: http://www. cimm-icmm.org/page/france/vaccinationFR/ABSTRACT.pdf

23. Richard V, Haus R, Verret C, Molinier S, Hugard L, Nicand E, et al. [Impact and forecasting of hepatitis $A$ immunization in French armed forces, 1990-2004]. Rev Epidemiol Sante Publique. 2006;54(5):433-41. French.

24. Bégaud B, Evreux JC, Jouglard J, Lagier G. [Imputation of the unexpected or toxic effects of drugs. Actualization of the method used in France]. Therapie. 1985;40(2):111-8. French. 